\title{
Oferta e demanda na atenção domiciliar em saúde
}

\author{
Supply and demand in home health care
}

\author{
Patrícia Pinto Braga ${ }^{1,2}$ \\ Roseni Rosângela de Sena ${ }^{1.3}$ \\ Clarissa Terenzi Seixas ${ }^{1,4}$ \\ Edna Aparecida Barbosa de Castro ${ }^{1,5}$ \\ Angélica Mônica Andrade 1,3 \\ Yara Cardoso Silva ${ }^{1,3}$
}

${ }^{1}$ Núcleo de Estudos e Pesquisas sobre Ensino e Prática em Enfermagem, Universidade Federal de São João del-Rei.R. Sebastião Gonçalves Coelho 400, Chanadour. 35501-296 Divinópolis MG Brasil. patricia_braga@ufsj.edu.br ${ }^{2}$ Faculdade de Enfermagem da Universidade Federal,

São João Del-Rei. São João del Rei MG Brasil.

${ }^{3}$ Escola de Enfermagem, Universidade Federal de Minas Gerais. Belo Horizonte MG Brasil.

${ }^{4}$ Faculdade de Enfermagem, Universidade do Estado do Rio de Janeiro. Rio de Janeiro RJ Brasil.

${ }^{5}$ Faculdade de Enfermagem, Universidade Federal de Juiz de Fora. Juiz de Fora MG Brasil.
Abstract The changes in the demographic and epidemiologic profiles of the Brazilian population and the need to rethink the health care model have led many countries like Brazil to consider Home Care (HC) as a care strategy. However, there is a gap between the supply of HC services, the demand for care and the health needs manifested by the population. Thus, this article analyzes scientific output regarding the status of the relation between supply, demand and the needs related to home health care. This work is based on an integrative review of the literature in the following databases: Cumulative Index to Nursing and Allied Health Literature (CINAHL), Latin America and the Caribbean Literature on Health and Science (Lilacs), Medical Literature Analysis and Retrieval System Online (Medline) and Web of Science. Despite the fact that few articles refer to the issue in question, there is evidence indicating that health demands and needs are seldom taken into account either in a quantitative or qualitative approach when developing the organization of HC services. The analysis would indicate that there is a national and international deficit in the supply of HC services considering the demand for health care and needs currently prevailing.

Key words Home care services, Organizational models, Public health policy
Resumo As mudanças no perfil demográfico e epidemiológico da população brasileira e a necessidade de repensar o modelo de atenção em saúde têm levado diversos países, como o Brasil, a pensar a Atenção Domiciliar (AD) como estratégia de atenção. Porém, identifica-se uma lacuna entre a oferta de serviços de AD, as demandas por cuidados e as necessidades em saúde apresentadas pela população. Assim, este artigo analisa as produções científicas ${ }_{2}$ quanto à configuração das relações entre oferta, demanda e necessidades relacionadas à atenção domiciliar em saúde. Trata-se de uma revisão integrativa da literatura nas bases de dados: Cumulative Index to Nursing and Allied Health Literature (CINAHL), Literatura Latino Americana e do Caribe em Ciências da Saúde (Lilacs), Medical Literature Analysis and Retrieval System Online (Medline) e Web of Science. Apesar de poucos artigos se aterem a essa questão, há evidências de que as demandas e as necessidades em saúde pouco têm sido levadas em conta para pensar a organização dos serviços de $A D$, tanto quantitativa quanto qualitativamente. A análise permite inferir que há um déficit na oferta de serviços de $A D$ no contexto nacional e internacional considerando as demandas por cuidados e necessidades de saúde que se apresentam.

Palavras-chave Serviços de assistência domiciliar, Modelos organizacionais, Politicas públicas de saúde 


\section{Introdução}

A utilização do domicílio como espaço de cuidado em saúde é uma prática que tem sua origem em tempos remotos: todos realizamos alguma forma em nossas casas. No entanto, a institucionalização dessa prática e a profissionalização do cuidado no domicílio surgem nos Estados Unidos em 1947, motivadas pela necessidade de liberar leitos hospitalares e de criar um ambiente mais favorável à recuperação dos pacientes ${ }^{1}$. Nos anos que se seguiram, sobretudo a partir da década de $1970^{2}$, observou-se o aumento dos serviços de atenção domiciliar (AD) sob as mais diversas modalidades na maior parte das sociedades ocidentais, movimento que apresenta tendência à aceleração na atualidade.

O incremento da $\mathrm{AD}$ no cenário mundial vem acompanhando as mudanças demográficas e epidemiológicas e está relacionado à racionalização da utilização dos leitos hospitalares, redução dos custos da assistência para os sistemas de saúde e pelo estabelecimento de uma lógica de cuidado embasada na humanização $0^{3-5}$. Destaca-se, contudo, que esta expansão não acontece de forma homogênea no cenário mundial, haja vista as diferenças no contexto político e na configuração dos sistemas de saúde entre os países, bem como as especificidades sociais, econômicas e culturais que influenciam sobremaneira o modo de operar e ofertar o cuidado domiciliar. Essas diferentes realidades influenciam também os arranjos assistenciais e as modalidades de Atenção Domiciliar ofertadas no Brasil e no mundo. Nessa perspectiva, a análise sobre oferta e demanda no campo da $\mathrm{AD}$ exige considerar a heterogeneidade existente nesse campo e as especificidades locais.

No Brasil, a primeira experiência de cuidado profissional no domicílio de que se tem notícia data de 1949, realizada pelo Serviço de Assistência Médica Domiciliar e de Urgência (SAMDU), vinculado ao Ministério do Trabalho e incorporado ao Instituto Nacional de Previdência Social (INPS) em 19676. À partir de então, observou-se a expansão dos serviços de $\mathrm{AD}$ no Brasil, especialmente da década de 1990 em diante. Essa expansão esteve associada, sobretudo, ao aumento da demanda por cuidados de certos grupos populacionais, em especial aqueles acometidos por doenças crônico-degenerativas, e concentrou-se na iniciativa privada e nos grandes centros urbanos ${ }^{4}$.

Reconhecendo essas mudanças e a necessidade de políticas públicas que viessem subsidiar a organização das diversas modalidades da $\mathrm{AD}$ no Brasil, foi publicada a Portaria Ministerial $\mathrm{n}^{\circ}$. 2.416 de 1998, que definia requisitos para o credenciamento de hospitais e critérios para a modalidade de internação domiciliar no SUS7. Outro marco legislativo importante foi a sanção pelo Ministério da Saúde, em abril de 2002, da Lei no 10.424 que acrescentava à Lei Federal $n^{\circ}$ 8.080/90, o atendimento e a internação domiciliar no Sistema Único de Saúde (SUS) ${ }^{8}$. Registrase então o incremento das discussões acerca de uma política e de um programa específicos que incorporem e regulamentem o cuidado no domicílio no contexto do SUS, procurando definir e delimitar suas diferentes modalidades, definir mecanismos específicos para garantir a regulação e o financiamento da AD no âmbito do SUS, entre outros. Esse esforço culminou na publicação de uma série de portarias no período de 2006 a 2013 e no lançamento do Programa Melhor em Casa que instituiu a Política Nacional de Atenção Domiciliar no SUS em 20119.

A incorporação da AD no SUS pretendeu garantir o cumprimento dos princípios e diretrizes constitucionais de universalidade, integralidade, equidade, participação comunitária, descentralização, regionalização e hierarquização e representou uma tentativa de resposta às demandas crescentes por outras formas de cuidado não hospitalares no país. A demanda por essas modalidades de cuidado domiciliar ganhou impulso com o aumento da visibilidade do processo de envelhecimento populacional brasileiro ${ }^{10}$, que tem gerado um aumento no número de idosos acometidos por doenças crônico-degenerativas, com perda de sua independência e com necessidade de cuidados permanentes, colocando em cheque o modelo de atenção à saúde que tem o hospital como local prioritário do cuidado ${ }^{11}$.

Outras demandas emergentes em saúde no cenário brasileiro também são igualmente relevantes e elegíveis para o cuidado no domicílio, tais como as de crianças e adultos acometidos com doenças crônicas classificadas como raras ${ }^{12}$. Para se ter uma ideia, $31,3 \%$ da população sofre de algum problema crônico de saúde e cerca de $6 \%$ dos brasileiros chegam a ter concomitantemente até três doenças crônicas ${ }^{13}$.

Entretanto, embora se reconheça a evolução em relação à legislação que regulamenta a $\mathrm{AD}$ no país, sua aposta não tem sido em seu potencial de substitutividade no SUS, mas, sobretudo no seu caráter complementar ${ }^{14}$. Assim, a exigência é mais do que uma reorganização dos campos de prática e dos locais para a realização do cuidado, mas inclui a mudança de concepção sobre os modos de planejar e realizar o cuidado em saú- 
$\mathrm{de}^{15}$. Nessa perspectiva há que se repensar as tecnologias de que lançamos mão para a produção do cuidado e considerar que elas devem buscar responder às demandas e necessidades quando se oferta um serviço de AD.

Esta oferta além de exigir especificidades relativas ao processo de trabalho pode também ser atravessada por outros aspectos como os critérios de elegibilidade para a AD. Esta realidade pode ser identificada no contexto do Brasil e do Canadá, por exemplo, uma vez que as condições adequadas do domicílio para a prestação de serviços domiciliares pode ser uma das condições para a oferta da AD nestes países. Nestes casos, entende-se que há dificuldade de ofertar a atenção domiciliar em casos de pacientes que residem em condições precárias pela inviabilidade da adoção dos protocolos requeridos ${ }^{16}$.

Pode-se indicar que mais do que a insuficiência quantitativa de serviços e programas de $\mathrm{AD}$ ofertados, há um descompasso entre a lógica sob a qual se organiza essa oferta - a da racionalização de custos - e a demanda e necessidades de saúde da população. Ou seja, enquanto os serviços ofertados vêm responder às necessidades de gestão, de se encontrar a melhor alocação entre recursos disponíveis e demandas iminentes e, ainda que atenda a certas demandas de cuidado, essa oferta não incorpora efetivamente as necessidades de saúde na sua agenda.

O reconhecimento da demanda potencializa o atendimento das necessidades de saúde do indivíduo que se apresenta $\mathrm{e}^{17}$ deve seguir um movimento inverso da lógica biomédica, centrada na doença, passando para uma ótica centrada no usuário, o que significa tratar o usuário como sujeito social portador de aspirações, desejos e histórias de vida e, portanto, que deve participar ativamente da definição do seu projeto terapêutico.

A análise do contexto de atenção à saúde das últimas décadas, como expresso anteriormente, sinaliza mudanças no perfil de morbidade da população que exigem estratégias de atenção à saúde de caráter contínuo, como é percebido em programas de AD no Brasil e no mundo. Associado a isso, percebe-se que a $\mathrm{AD}$ tem sido marcada por uma lógica de racionalização de custos e, também, de reestruturação produtiva do cuidado.

A partir deste cenário e considerando que, mesmo após a criação das políticas de $\mathrm{AD}$ no SUS, a oferta desses serviços ainda não é capaz de responder à demanda de cuidado que atenda às necessidades de saúde da população, elaboramos a seguinte questão norteadora que desencadeou o desenvolvimento desta revisão integrativa: Como se configuram as relações entre oferta e demanda de serviços e necessidades de saúde relacionadas à Atenção Domiciliar Nacional e Internacional?

Assim, este estudo tem como objetivo analisar as produções científicas da América Latina, Europa e Estados Unidos da América, quanto à configuração das relações entre oferta, demanda e necessidades de saúde relacionadas à $\mathrm{AD}$ em saúde.

A realização deste estudo justifica-se pela contribuição que trará na produção do conhecimento sobre o tema, em especial para repensar a lacuna existente entre oferta e demanda de serviços na $\mathrm{AD}$. Poderá contribuir também para a produção técnica científica sobre o tema e na organização de serviços.

\section{Metodologia}

Optou-se por uma revisão integrativa, por configurar um método de revisão mais amplo, permitindo a inclusão de estudos experimentais e não experimentais e favorecendo uma compreensão abrangente do tema. Este método tem a potencialidade de contribuir com conhecimentos resultantes de diferentes abordagens metodológicas para a necessidade de novas pesquisas, além de contribuir para a construção de políticas em saúde ${ }^{18}$.

Desta forma, foram cumpridas as seguintes etapas: identificação do tema; formulação de uma questão norteadora; busca e seleção da literatura; categorização e avaliação dos estudos; e apresentação da revisão ${ }^{19}$.

Para busca e seleção da literatura definiramse as seguintes bases de dados: Cumulative Index to Nursing and Allied Health Literature (CINAHL), Literatura Latino Americana e do Caribe em Ciências da Saúde (Lilacs), Medical Literature Analysis and Retrieval System Online (Medline) e Web of Science. Como critério de inclusão dos estudos, foi definido o período de publicação entre 2008 e 2013, trabalhos disponíveis eletronicamente na íntegra e trabalhos nos seguintes idiomas: inglês, espanhol e português.

As palavras-chave "home care services", "organizational models" e "health public policy" foram utilizadas de forma associada e, para a busca na base de dados Lilacs, os mesmos termos foram utilizados, sendo anteriormente traduzidos para a língua portuguesa.

$\mathrm{Na}$ busca inicial, foram encontrados 1.561 publicações. Pela leitura dos títulos e resumos, 
foi possível excluir os estudos em duplicidade nas diferentes bases de dados, os que não atendessem aos critérios de inclusão, aqueles que não atendiam ao tema proposto e aqueles aos quais não foi possível ter acesso na íntegra. Desses, foram selecionados 73 artigos que foram lidos na íntegra, dos quais 37 responderam à questão norteadora e, dessa forma, constituíram a amostra final desta revisão.

Para análise, os artigos incluídos foram lidos exaustivamente, o que permitiu a apropriação e a organização de seus conteúdos em tabelas de acordo com os seguintes itens: autoria, título, ano de publicação, periódico, objetivo, metodologia, nível de evidência científica, principais resultados, aspectos que apontam para as demandas por cuidados domiciliares e necessidades de saúde, aspectos que apontam para os serviços ofertados na $\mathrm{AD}$ e aspectos que sinalizam para a relação entre oferta, demanda e necessidades de saúde. Esta organização permitiu sistematizar a análise, o que favoreceu a apresentação dos resultados neste artigo.

Para classificação do nível de evidência $(\mathrm{NE})^{20}$ dos estudos optou-se pelo grupamento das publicações da seguinte forma: nível I - evidência obtida do resultado de metanálise de estudos clínicos controlados e com randomização; nível II - evidência obtida em estudo de desenho experimental; nível III - evidência obtida de pesquisas quase-experimentais; nível IV - evidências obtidas de estudos descritivos ou com abordagem metodológica qualitativa; nível $\mathrm{V}$ - evidências obtidas de relatórios de casos ou relatos de experiências; nível VI - evidências baseadas em opiniões de especialistas ou com base em normas ou legislação. Esta classificação permitiu revelar o perfil dos estudos desenvolvidos acerca do tema aqui investigado.

\section{Resultados e Discussão}

Em relação aos tipos de pesquisas incluídas, foram identificados vinte e dois estudos descritivos $(59,4 \%)$, quatro revisões de literatura (10,8\%), quatro relatos de experiências $(10,8 \%)$, dois estudos de reflexão $(5,4 \%)$, dois estudos randomizados $(5,4 \%)$, uma análise documental $(2,7 \%)$, uma metanálise $(2,7 \%)$ e um estudo quase experimental (2,7\%).

A análise dos tipos de estudo incluídos e seus respectivos níveis de evidências revelam que no campo da $\mathrm{AD}$, quando nos referimos à serviços ofertados, necessidades de saúde e demandas por cuidados, há o predomínio de estudos descritivos $(59,4 \%)$, ou seja, com nível de evidência IV, o que permite inferir que são temas ainda em exploração e que a expansão e o desenvolvimento de estudos como metanálises e pesquisas experimentais se fazem necessários.

A análise dos artigos incluídos nesta revisão permitiu revelar as seguintes categorias: Demandas e necessidades no campo da AD; Serviços de $\mathrm{AD}$ ofertados; Relação entre oferta, demanda e necessidade de saúde no contexto da AD.

\section{Demandas e Necessidades no campo da Atenção Domiciliar}

O envelhecimento da população no Brasil e no mundo está ancorado nos avanços tecnológico-assistenciais que permitiram o aumento da expectativa de vida ${ }^{4,21-31}$. Nesse cenário, $o$ surgimento de um novo perfil de demandas por cuidados específicos e de novas necessidades de saúde é uma constatação da maioria dos estudos incluídos nesta revisão. Assim, pessoas vivendo com doenças crônicas complexas, como Insuficiência Cardíaca Congestivaa ${ }^{21,32-34}$, Doença Pulmonar Obstrutiva Crônica grave ${ }^{35}$, Pacientes sob Ventilação Mecânica ${ }^{36}$, Paralisia Cerebral Infanti $^{35,37}$, e, ainda, aqueles com doenças degenerativas avançadas, têm sido apontadas como demandantes de cuidados longitudinais, contínuos e de longa duração $0^{38}$.

Esse novo perfil é fortemente impactado pela maior dependência para as atividades da vida diária - tais como alimentação, banho, deslocamento e autocuidado - dessa população, o que tem exigido rearranjos familiares, a contratação de pessoas e/ou de diferentes serviços para atendimento às necessidades cotidianas desta população $0^{21,32,33,35}$. Nesse contexto, a Atenção Domiciliar tem se mostrado como uma opção para reduzir os gastos e para permitir a elaboração de planos de cuidado de forma compartilhada com as famílias ${ }^{21,28,36}$.

Um estudo americano indica que o número de idosos confinados no domicílio que poderiam se beneficiar de $\mathrm{AD}$ é significativamente maior quando comparado ao de trabalhadores qualificados e desejosos de trabalhar com essa modalidade $^{23}$, e que essa assimetria deverá aumentar consideravelmente em um futuro próximo - a previsão é de que, no horizonte 2050, o número de trabalhadores já será insuficiente para atender à demanda por cuidados apresentada pela população idosa nos EUA, indicando a necessidade de formar pessoas para o trabalho no domicílio. No 
que tange especificamente aos cuidadores domiciliares, tanto no panorama nacional como no internacional, os estudos sinalizam para a presença do cuidador, seja ele formal ou informal, como uma prerrogativa para que a $\mathrm{AD}$ se concretize. Adotamos a concepção de que cuidador formal entendido como aquele capacitado tecnicamente e treinado para o cuidado no domicílio. Já o cuidador informal, papel geralmente assumido por familiares, amigos ou vizinhos, é aquele que não possui vínculo formal de trabalho e/ou formação técnica para tal.

Outros estudos mostram que o cuidado domiciliar de pacientes complexos já tem exigido a qualificação e especialização dos profissionais para o atendimento das demandas específicas de cuidados que este perfil de paciente apresen$\mathrm{ta}^{21,35-36}$. Esses dados são corroborados por diversos estudos internacionais que apontam para uma escassez global de trabalhadores formais e informais para o trabalho no domicílio, que vem sendo parcialmente suprida, nos países desenvolvidos, através da flexibilização das políticas migratórias para trabalhadores da saúde $\mathrm{e}^{39-41}$.

Além da evidência de déficit de cuidadores formais, estudos tem alertado para a necessidade de se cuidar dos cuidadores informais, considerando a sobrecarga que advém ao assumir os cuidados cotidianos, as situações de estresse que envolvem o cuidar de forma continuada, as negociações e os abandonos de emprego e a administração dos gastos com o cuidado que assumem $^{4,26,27,42-45}$, o que podemos pensar em termos de oportunidades de vida perdidas. Dentre os processos de adoecimento que aparecem com mais frequência para os cuidadores informais ao assumir os cuidados de uma pessoa, destacamse a ansiedade, a depressão, a fadiga, a perda de sono e os problemas musculoesqueléticos ${ }^{27,44}$. A análise sinaliza para a necessidade de se pensar formas de apoiar os cuidadores informais no seu trabalho, na garantia de uma formação mínima, assegurando formas de compensação por eventuais perdas financeiras e na criação de espaços de escuta e de apoio, produzindo dispositivos de educação permanente.

A análise dos artigos também indica que a Atenção Domiciliar tem uma especificidade do cuidado e, consequentemente, do processo de trabalho em razão do perfil dos pacientes, mas também da lógica que opera no domicílio. Isso implica em uma necessidade de formação diferenciada para responder à uma demanda crescente. Desse modo, compreendemos a necessidade de se apostar na $\mathrm{AD}$ como uma possibilidade na perspectiva da substitutividade ${ }^{4}$, sob risco de não atender às necessidades de saúde da população e prejudicando, assim, a qualidade de vida destes usuários. A partir dessas constatações, fica evidente que a necessidade quantitativa e qualitativa de trabalhadores para o cuidado no domicílio deve constituir uma prioridade na agenda política brasileira para a sustentabilidade de um modelo de atenção em consonância com as mudanças demográficas e epidemiológicas e a fim de garantir a atenção às pessoas nos seus domicílios de acordo com a complexidade do cuidado demandado.

Em um estudo de coorte de dez anos, desenvolvido em Milão, Itália ${ }^{35}$, a Atenção Domiciliar mostrou-se benéfica para pacientes com Doença Pulmonar Obstrutiva Crônica (DPOC) grave. A pesquisa identificou que o grupo de pacientes com DPOC atendidos por serviços de $\mathrm{AD}$ apresentou menor mortalidade do que o grupo atendido na modalidade tradicional. Evidenciou também que os pacientes em $\mathrm{AD}$ apresentaram menores taxas de crises agudas e de piora clínica, procuraram menos os serviços de emergência e apresentaram menores taxas de reinternação. Um estudo sobre necessidades de saúde não atendidas desenvolvido na província de Quebec no Canadá ${ }^{46}$, revelou que quando o paciente está sendo atendido em alguma modalidade de $\mathrm{AD}$, $35 \%$ de suas necessidades não são atendidas, ao passo que quando ele não está inserido em um serviço de $\mathrm{AD}$, esse percentual quase duplica, chegando a $67 \%$. A análise permite inferir que mesmo em situações de agravos complexos à saúde, o cuidado ofertado na modalidade de $\mathrm{AD}$ tem sinalizado para melhorias na qualidade da assistência prestada e responde melhor às necessidades de saúde apresentadas. Um aspecto relevante a ser considerado nesta análise é que o Canadá possui uma organização e uma oferta de serviços de Atenção Domiciliar que está intensamente articulado ao sistema de saúde local, o que permite uma cobertura e acesso a este serviço de destaque no cenário internacional.

Destaca-se, ainda, que as condições socioeconômicas do Canadá e do Brasil são bastante diferenciadas, assim como a estrutura e a organização dos serviços de saúde nos dois países. Um estudo comparando a proposta de AD no âmbito da atenção básica no Brasil com o modelo adotado pelo Canadá revelou as diferenças em relação aos indicadores sociosanitários, a exemplo do Índice de Desenvolvimento Humano (IDH), no qual o Canadá entre 1994 e 1997 encontrava-se em 1º lugar e o Brasil em 69º, em 2002. Quanto ao sis- 
tema de saúde, o Canadá conta com um sistema de financiamento predominantemente público e o setor privado é complementar, enquanto no Brasil o financiamento é misto, ou seja, público -privado, havendo uma disputa entre os mesmos, inclusive de modelos de atenção ${ }^{16}$.

Nesta perspectiva, as afirmativas da análise tecida nesta revisão expressam realidades locais que possuem influência de aspectos políticos e organizacionais no campo da saúde e são também fortemente determinadas por aspectos culturais, sociais e econômicos. Assim, não se trata de fazermos generalizações, mas de sinalizar os diferentes arranjos de oferta e demanda na AD.

A partir da revisão realizada, pode-se afirmar que uma grande diversidade e multiplicidade de demandas e necessidades se apresentam no âmbito da AD. Os artigos analisados revelam um conjunto de condições de saúde e de situações que se configuram como demandas passíveis de serem atendidas no domicílio, como Doenças Crônicas (Insuficiência Cardíaca Crônica, Insuficiência Renal Crônica, Diabetes); Sequelas de Acidente Vascular Cerebral (AVC); Reabilitação Cardiovascular; Pacientes em situação de risco de abandono de tratamento de Tuberculose; Atendimento a pacientes com HIV/AIDS; Acompanhamento de idosos com quadro de depressão; Portadores de feridas e lesões de pele; Suporte Ventilatório (pacientes com DPOC); Cuidados Paliativos; Atendimento a acamados crônicos; Cuidados a jovens e crianças com deficiência múltipla; Crianças nascidas prematuras e de baixo peso; Doenças agudas como infecção urinária, infecções respiratórias para antibioticoterapia endovenosa; Gestantes e puérperas (mulheres no período pós-parto); e agravos de natureza psicossocial.

A análise sinaliza, ainda, que a demanda no âmbito da $\mathrm{AD}$ vai se dando em diferentes dimensões, como a prevenção de agravos, a recuperação e a reabilitação da saúde e também com tentativas de promoção da saúde ${ }^{26}$. Além disso, identifica-se que, apesar de o público ser composto predominantemente por idosos, outras faixas etárias também têm sido atendidas em serviços de AD. Esses dados revelam a diversidade de perfis, condições e situações passíveis de atendimento domiciliar, o que olhado pelo lado das necessidades de saúde, evidencia quão representativo é o rol de demandas reprimidas da qual não se tem, ainda, a real dimensão.

Dessa forma, constata-se que muitas das necessidades em saúde vêm sendo negligenciadas no cenário brasileiro, o que parece revelar tanto uma insuficiência das instâncias gestoras de proporcionar condições aos serviços para dar conta dessa multiplicidade e de situações passíveis de serem atendidas no domicílio - relacionadas à força de trabalho preparada para ocupar esses espaços, às condições de financiamento para estruturar esses serviços, etc, - como uma aposta ético-política em um modelo de atenção onde a $\mathrm{AD}$ não constitui um espaço privilegiado para o cuidado em saúde, ocupando um lugar periférico no rol de ofertas e onde apenas situações muito pontuais se encaixam.

\section{Serviços de Atenção Domiciliar ofertados}

Os cenários nacional e internacional contemporâneos, conforme citado anteriormente, caracterizam-se por um aumento da demanda de cuidados a pacientes com condições crônicas e requerem novas modalidades de cuidado que possam efetivamente atender às necessidades de saúde que se apresentam. A análise da relação entre a demanda e a oferta de serviços de Atenção Domiciliar em cenários onde operam sistemas de saúde com configurações diversas, evidencia as principais motivações que desencadearam a implantação de serviços de $\mathrm{AD}$, podendo essas ser assim sintetizadas: a desospitalização e a liberação de leitos hospitalares; a desinstitucionalização de idosos em instituições de longa permanência; a racionalização dos gastos; a humanização da assistência; a integralidade da atenção; o interesse em evitar a internação hospitalar; e a reestruturação dos modelos de atenção vigentes ${ }^{24,26,28,30,34,47-50}$. O levantamento dessas motivações permite constatar que, tanto no cenário internacional quanto no Brasil, as motivações para a implantação dos serviços são semelhantes e têm em vista tanto aspectos econômicos, quanto questões que revelam uma preocupação com a qualidade de vida e do cuidado que é prestado no domicílio. Esses dados são corroborados por recente publicação do Ministério da Saúde em parceria com a Organização Pan-Americana de Saúde que traz dados comparativos entre as estratégias e os serviços de Atenção Domiciliar adotados em alguns países selecionados ${ }^{51}$.

Os artigos analisados mostram que há uma diversidade na oferta de serviços de $\mathrm{AD}$, a saber: serviços de gerenciamento de casos de doenças crônicas, como as doenças pulmonares, cardiovasculares, a AIDS e a diabetes ${ }^{29,45,48}$; serviços de reabilitação no domicílio $5,29,35,38,50$; serviços de monitoramento telefônico para pacientes em pós-cirúrgico e acompanhamento terapêuti- 
$\operatorname{co}^{27,46}$; serviços operando na modalidade de telemedicina ou telessaúde (que utiliza instrumentos de vídeoconferência para atendimento a pacientes com diabetes e idosos com depressão, por exemplo) $)^{31,48}$; serviços para acompanhamento de situações agudas de saúde como infecções urinárias e pulmonares; assistência no domicílio à pessoas em situação de vulnerabilidade social ${ }^{4}$, etc.

Dentre as diferentes modalidades de $\mathrm{AD}$ identificadas nesta revisão, ressaltam-se as estratégias de assistência presencial e à distância. $\mathrm{Na}$ modalidade à distância, a educação em saúde surge como um elemento-chave. Na modalidade presencial, ou seja, na qual a equipe precisa se deslocar ao domicílio periodicamente, predomina o desenvolvimento de diferentes procedimentos, de "intervenções diretas sobre os corpos" dos usuários e esta é a modalidade predominante no contexto brasileiro.

A partir da análise dos dados, buscou-se categorizar a $\mathrm{AD}$ em modalidades levando em conta os tipos de serviços ofertados e não a intensidade ou complexidade tecnológica do cuidado, conforme mostrado no Quadro 1.

Na modalidade de Atenção Domiciliar à saúde, para os serviços descritos nos artigos analisados, que são prestados por equipes multiprofissionais, são fornecidos os insumos necessários para que o cuidado seja realizado nesse local, assim como exames, transporte e suporte inter- disciplinar quando estes são necessários e estão disponíveis $32,36,38,45,48,52$.

A análise permitiu compreender que, dentre as modalidades de $\mathrm{AD}$ apresentadas nos artigos, a modalidade de Atenção Domiciliar à saúde no domicílio predomina no cenário nacional em detrimento das outras modalidades apresentadas no Quadro 1. Esse achado apresenta um leque de possibilidades de $\mathrm{AD}$ que podem ser pensadas para a realidade brasileira. A oferta de serviços aliada à articulação entre diferentes setores e serviços também apresentou-se como estratégia que fortalece a integralidade da $\mathrm{AD}$ e a qualidade do cuidado prestado ${ }^{28,36}$.

Também foi possível identificar nos artigos analisados, a possibilidade de construção compartilhada do plano terapêutico entre usuários, familiares e profissionais como uma potencialidade de diversos serviços de $A D^{21,25,46}$. Outra evidência da análise é que a $\mathrm{AD}$ exige de maneira mais evidente das equipes, o raciocínio clínico a fim de avaliar as situações que porventura se apresentem, considerando as dificuldades implicadas na remoção do usuário para realização de exames. Essas constatações vêm reafirmar o potencial da Atenção Domiciliar como reordenadora do cuidado, ao ter como elementos centrais do seu processo a escuta do outro, o reconhecimento do conhecimento informal de cuidadores, familiares e usuários, em suma, das tecnologias leves ${ }^{53}$.

Quadro 1. Modalidades de serviços de Atenção Domiciliar ofertados.

\begin{tabular}{|l|l|}
\hline \multicolumn{1}{|c|}{ Modalidade } & \multicolumn{1}{c|}{ Descrição } \\
\hline $\begin{array}{l}\text { Atenção Domiciliar à Saúde no } \\
\text { domicílio - o domicílio como lócus } \\
\text { do cuidado }\end{array}$ & $\begin{array}{l}\text { Nesta modalidade a equipe de profissionais de saúde realiza a } \\
\text { intervenção no domićlio, com submodalidades de acordo com a } \\
\text { complexidade do cuidado e as tecnologias envolvidas (Cuidados } \\
\text { primários à saúde, internação domiciliar, etc.) }\end{array}$ \\
\hline $\begin{array}{l}\text { Atenção Domiciliar } \\
\text { Institucionalizada - instituições que } \\
\text { ofertam cuidados (Hospice) }\end{array}$ & $\begin{array}{l}\text { Modalidade predominante na Europa, na qual o setor público ou } \\
\text { privado monta equipamentos que se assemelham ao ambiente } \\
\text { hospitalar. Estes serviços contam com profissionais que atendem às } \\
\text { demandas por cuidados apresentadas pelos usuários que passam a } \\
\text { residir temporariamente ou permanentemente nestes locais. }\end{array}$ \\
\hline $\begin{array}{l}\text { Atenção Domiciliar à Distância } \\
\text { - Telemonitoramento; } \\
\text { vídeoconferência pela internet. }\end{array}$ & $\begin{array}{l}\text { Modalidade que utiliza o telefone ou a internet como recurso para } \\
\text { intervir. Há predominantemente ações de educação em saúde e a } \\
\text { maioria dos serviços nesta modalidade são de prevenção. }\end{array}$ \\
\hline $\begin{array}{l}\text { Assistência no Domićlio às } \\
\text { demandas de cunho social }\end{array}$ & $\begin{array}{l}\text { Modalidade de assistência social às famílias em situação de } \\
\text { vulnerabilidade social e/ou econômica, como idosos acamados e sem } \\
\text { possibilidades de fazer suas próprias compras, por exemplo. }\end{array}$ \\
\hline
\end{tabular}




\section{Relação entre oferta, demanda e necessidade de saúde no contexto da Atenção Domiciliar}

A análise permitiu identificar um déficit na oferta de serviços de AD mediante a demanda que se apresenta no cenário nacional e internacional $^{23,26,27,38,49,54,55}$.

No Canadá, por exemplo, país que apresenta a AD consolidada na rede de serviços do seu sistema de saúde, identificamos que, mesmo tendo uma organização favorável para ofertar serviços de $\mathrm{AD}$, têm sido necessárias contratações na iniciativa privada (pelos prestadores de cuidados domiciliar ou pela família) para atender às necessidades que se apresentam ${ }^{42}$.

No Brasil, há um déficit na oferta de serviços de $\mathrm{AD}$ em geral. Isso é evidente, por exemplo, no que tange a serviços que ofereçam cuidados paliativos aos seus usuários, conforme dados do Observatório Internacional de Cuidados de Final da Vida (IOLEC), e este dado torna-se relevante quando reconhecemos que a neoplasia é a segunda maior causa de morte no Brasil ${ }^{52}$. Esse déficit é dificilmente quantificável, sobretudo pela impossibilidade de se estabelecer critérios aplicáveis em larga escala para avaliar as possibilidades e as vantagens de inclusão de pacientes em serviços de $\mathrm{AD}$ e a demanda reprimida.

Outra questão apontada na análise é que o aumento na oferta de serviços de $\mathrm{AD}$ não atenderá todas as necessidades/demandas da população, mas são necessárias estratégias para integrar serviços numa rede de cuidados a fim de potencializar a integralidade e a resolutividade ${ }^{46}$. Em um estudo realizado no Reino Unido, sinaliza-se para a insustentabilidade financeira dos serviços de $\mathrm{AD}$, considerando o aumento da demanda por esta modalidade de cuidado em um contexto de declínio de pessoas em idade "ativa", diminuindo a oferta de trabalhadores/cuidadores ${ }^{25}$.

A análise permite inferir que estudos sobre a relação entre a oferta de serviços, demandas por cuidados e necessidades de saúde são ainda incipientes para ampliar a análise quantitativa da necessidade de $\mathrm{AD}$ nos países.

\section{Considerações Finais}

As mudanças contemporâneas nos perfis demográficos e epidemiológicos das populações permitem reflexões sobre a inadequabilidade dos modelos e das modalidades de atenção que têm orientado a organização dos sistemas de saúde, tanto no cenário nacional quanto no internacional. Nesse contexto, a demanda por cuidados no domicílio tem aumentado consideravelmente em todos os países, independente das diferenças socioeconômicas e de organização dos serviços de saúde. A análise dos artigos aponta para o déficit de cuidadores frente a demanda crescente, evidenciando a necessidade de formação destes profissionais para atendê-la e atuar nas diversas questões envolvendo o cuidador, um ator central na atual configuração dos serviços de AD.

Ainda que a demanda por $\mathrm{AD}$ não esteja mensurada, a expansão da oferta desses serviços não tem acompanhado a demanda, tanto quantitativa quanto qualitativa, nas suas diversas modalidades. No Brasil, a revisão evidenciou apenas uma modalidade de Atenção Domiciliar: a AD em saúde no domicílio - este como locus do cuidado, o que revela uma necessidade de se olhar para o que se tem feito em outros países como possibilidade para organização desse serviço no Brasil.

$\mathrm{Na}$ reorganização da oferta, também fica evidente a importância de se repensar os critérios para a inclusão de pacientes na $\mathrm{AD}$, uma vez que a compreensão de quais perfis de pacientes poderiam ou não ser incluídos em serviços é variável de acordo com a concepção que se tem da $\mathrm{AD}$ - como modalidade complementar ou substitutiva dentro da rede de cuidados em saúde. A análise da $\mathrm{AD}$ atua como uma estratégia para a substitutividade que opera em uma nova lógica de atenção que avance para o cuidado de qualidade em saúde no Brasil. Para tanto é necessária a orientação das práticas de saúde incluindo as necessidades de cuidados no domicílio, com reorganização da oferta da Atenção Domiciliar e, consequentemente, a organização das redes de atenção à saúde.

Ademais, torna-se relevante a realização de estudos que relacionem as motivações para a criação de serviços de $\mathrm{AD}$ com o tipo de modalidade a partir da decisão de implantação, a fim de analisar a potencialidade desses equipamentos em responder - ou não - às necessidades de saúde da população que atende. 


\section{Colaboradores}

PP Braga, RR Sena, CT Seixas, EAB Castro, AM Andrade e YC Silva colaboraram igualmente na elaboração do artigo.

\section{Agradecimentos}

As autoras agradecem ao CNPq e FAPEMIG pelo apoio ao projeto de pesquisa "Atenção domiciliar em saúde: efeitos e movimentos na oferta e demana no SUS", que disparou a construção deste texto.

\section{Referências}

1. Ramallo VJG, Tamayo MIP. Historia de la hospitalización a domicilio. In: Glez MDD, coordinador. Hospitalización a domicilio. Madri: Hoechst Marion Rousse; 1998. p. 13-22.

2. Mendes EV. Os grandes dilemas do SUS. Salvador: Casa da Qualidade Editora; 2001.

3. Silva KL, Sena R, Leite JCA, Seixas CT, Gonçalves AM. Internação domiciliar no Sistema Único de Saúde. Rev Saude Publica 2005; 39(3):391-397.

4. Feuerwerker LCM, Merhy EE. A contribuição da atenção domiciliar para a configuração de redes substitutivas de saúde: desinstitucionalização e transformação de práticas. Rev Panam Salud Publica 2008; 24(3):180188.

5. Kerber NPC, Kirchhof ALC, Cezar-Vaz MR. Considerações sobre a atenção domiciliária e suas aproximações com o mundo do trabalho na saúde. Cad Saude Publica 2008; 24(3):485-493.

6. Mendes Júnior WV. Assistência domiciliar: uma modalidade de assistência para o Brasil[dissertação]. Rio de Janeiro: Universidade Estadual do Rio de Janeiro; 2000.

7. Brasil. Portaria no 2.416 , de 23 de março de 1998. Estabelece requisitos para credenciamento de hospitais e critérios para realização de Internação Domiciliar no SUS. Diário Oficial da União 1998; 26 mar.

8. Brasil. Lei no 10.424, de 14 de abril de 2002. Acrescenta capítulo e artigo à Lei no 8.080 , de 19 de setembro de 1990, que dispõe sobre as condições para a promoção, proteção e recuperação da saúde, a organização e o funcionamento de serviços correspondentes e dá outras providências, regulamentando a assistência domiciliar no Sistema Único de Saúde. Diário Oficial da União 2002; 16 abr.

9. Brasil. Portaria MS/GM n 2.029 , de 24 de agosto de 2011. Institui a Atençãono âmbito do Sistema Único de Saúde (SUS). Diário Oficial da União 2011; 25 ago.

10. Brasil. Lei n. 10.741, de 1 de outubro de 2003. Dispõe sobre o Estatuto do Idoso e dá outras providências. Diário Oficial da União 2003; 3 out.

11. Karsch UM. Idosos dependentes: famílias e cuidadores. Cad Saude Publica 2003; 19(3):861-866.

12. Brasil. Portaria no 199, de 30 de janeiro de 2014. Institui a Política Nacional de Atenção Integral às Pessoas com Doenças Raras, aprova as Diretrizes para Atenção Integral às Pessoas com Doenças Raras no âmbito do Sistema Único de Saúde (SUS) e institui incentivos financeiros de custeio. Diário Oficial da União 2014; 31 jan.

13. Instituto Brasileiro de Geografia e Estatística (IBGE). Diretoria de Pesquisas Coordenação de Trabalho e Rendimento. Orçamento e Gestão. Rio de Janeiro: IBGE; 2010 .
14. Silva KL, Sena R, Leite JCA, Seixas CT, Gonçalves AM. Atenção domiciliar no Brasil: avanços e desafios para mudança no modelo tecnoassistencial em saúde. SIIC Salud 2007; 18:1.

15. Andrade AM, Brito MJM, Silva KL, Montenegro LC, Caçador BS, Freitas LFC. Organização das redes de atenção à saúde na perspectiva de profissionais da atenção domiciliar. Rev Gaúcha Enferm 2013; 34(2):111-117. Rehem TCMSB, Trad LAB. Assistência domiciliar em

16. saúde: subsídios para um projeto de atenção básica brasileira. Cien Saude Colet 2005;10(Supl.):231-242.

17. Silva JPV, Pinheiro R, Machado FRS. Necessidades, demanda e oferta: algumas contribuições sobre os sentidos, significados e valores na construção da integralidade na reforma do setor saúde. Saúde em Debate 2003; 27(65):234-242.

18. Whittemore R, Knafl K. The integrative review: updated methodology. J Adv Nurs 2005; 52(5):546-553.

19. Souza MT, Silva MD, Carvalho R. Integrative review: what is it? How to do it? Einstein 2010; 8(1 Pt 1):102-106.

20. Stetler CB, Brunell M, Giuliano KK, Morsi D, Prince L, Newell-Stokes V. Evidence-based practice and the role of nursing leadership. J Nurs Adm 1998; 28(7-8):45-53.

21. Konstam Marvin A, Greenberg B. Transforming Health Care Through the Medical Home: The Example of Heart Failure. J Card Fail 2009; 15(9):736-738.

22. Sørbye LW, Garms-Homolová V, Henrard J-C, Jónsson PV, Fialová D, Topinková E, Gambassi G. Shaping home care in Europe: The contribution of the Aged in Home Care project. Maturitas 2009; 62(3):235-242.

23. Hayashi J, DeCherrie L, Ratner E, Boling PA. Workforce Development in Geriatric Home Care. Clin Geriatr Med 2009; 25(1):109-120.

24. DeJonge KE, Taler G, Boling PA. Independence at Home: Community-Based Care for Older Adults with Severe Chronic Illness. Clin Geriatr Med 2009; 25(1):155-169.

25. Jagger C, Collerton JC, Davies K, Kingston A, Robinson LAE, Martin P, Zglinicki T, Martin-Ruiz C, James OFW; Kirkwood TBL, Bond J. Capability and dependency in the Newcastle $85+$ cohort study. Projections of future care needs. Geriatrics 2011; 11:21.

26. Kerber NPC, Kirchhof ALC, Cezar-Vaz MR. Considerações sobre a atenção domiciliária e suas aproximações com o mundo do trabalho na saúde.Cad Saude Publica 2008; 24(3):485-493.

27. Espinel-Bermúdez MC, Sánchez-García S, Juárez-Cedillo T, García-González JJ, Viveros-Pérez Á,Carmen GP. Impacto de un programa de atención domiciliaria al enfermo crónico en ancianos: calidad de vida y reingresos hospitalarios. Salud Publica Mex 2011; 53(1):17-25. 
28. Silva K, Sena RR, Seixas CT, Feuerwerker LCM, Merhy EE. Atenção domiciliar como mudança do modelo tecnoassistencial. Rev Saude Publica 2010; 44(1):166-176.

29. Morales-Asencio JM, Gonzalo-Jiméne E, Martin-Santos FJ, Morilla-Herrera JC, Celdráan-Mañas M, Carrasco AM, García-Arrabal JJ, Toral-López I.Effectiveness of a nurse-led case management home care model in Primary Health Care. A quasi-experimental, controlled, multi-centre study. BMC Health Services Research 2008; 8:193.

30. Marshall D, Howell D, Brazil K, Howard M, Taniguchi A. Enhancing family physician capacityto deliver quality palliative home care. Can Fam Physician 2008; 54(12):1703-1703.e7.

31. Sheeran T, Rabinowitz T, Lotterman J, Reilly CF, Brown S, Donehower P, Ellsworth E, Amour JL, Bruce ML. Feasibility and Impact of Telemonitor-Based Depression Care Management for Geriatric Homecare Patients. Telemed J E Health 2011; 17(8):620-626.

32. Pla B, Carlos A, Pedroso ERP. Assessment of the effectiveness of a home-based care program for patients coinfected with tuberculosis and human immunodeficiency virus after discharge from a reference hospital in South-Eastern Brazil. Braz J Infect Dis 2010; 14(6):594600.

33. Unroe KT, Greiner MA, Hernandez AF, Whellan DJ, Kaul P, Schulman KA, Peterson ED, Curtis LH. Resource Use in the Last 6 Months of Life Among Medicare Beneficiaries With Heart Failure. Am Med Assoc 2011; 171(3):196-203.

34. Sarela A, Whittaker MF, Ilkka KM. Service and Business Model for Technology Enabled and Home-based Cardiac Rehabilitation Programs. Annual International Conference of the Institute of Electrical and Electronics Engineers - IEEE; 2009.

35. Rizzi M, Grassi M, Pecis M, Andreoli A, Taurino AE, Sergi M, Fanfulla F. A Specific Home Care Program Improves the Survival of Patients With Chronic Obstructive Pulmonary Disease Receiving Long Term Oxygen Therapy. Arch Phys Med Rehabil 2009; 90(3):395-401.

36. Dybwik K, TollaliT, Nielsen EW,Brinchmann BS.Why does the provision of home mechanical ventilation vary so widely? Chron Respir Dis 2010; 7(2):67-73.

37. Silva ARA, Souza CV, Viana ME, Sargentelli G, Serpa MJA, Gomes MZR. Health care-associated infection and hospital readmission in a home care service for children. Am J Infect Control 2012; 40(3):282-283.

38. Stolt R, Blomqvist P, Windlad U. Privatization of social services: quality differences in Swedish elderly care. Soc Sci Med 2010; 72(4):560-567.

39. World Health Organization (WHO). The Solid Facts: Home Care in Europe. Milan: WHO Regional Office for Europe; 2008.

40. Lamura G, Polverini F. East to west: the migration of informal caregivers. Washington: AARP International; 2005.

41. Canadá. Live-in Caregivers, 2012. [acessado 2015 jun 10]. Disponível em: http://www.cic.gc.ca/english/work /caregiver/index.asp

42. Guerriere DN, Wong AYM, Croxford R, Leong VW, McKeever P, Coyte PC. Costs and determinants of privately financed home-based health care in Ontario, Canada. Health Soc Care Community 2008; 16(2):126136.
43. Hirakawa Y, Kuzuya M, Enoki H, Uemura K. Information needs and sources of family caregivers of home elderly patients.Arch Gerontol Geriatr 2011; 52(2):202205.

44. Williams AM, Eby JA, Crooks VA, Stajduhar K, Giesbrecht M, Vuksan M, Conhen C, Brazil K, Allan D. Canada's Compassionate Care Benefit: Is it na adequate public health response to addressing the issue of cargiver burden in end-of-life care. BMC Public Health $2011 ; 11: 335$.

45. Franco TB, Merhy EE. Atenção domiciliar na saúde suplementar dispositivo da reestruturação produtiva. Cien Saude Colet 2008; 13(5):1511-1520.

46. Dubuc N, Dubois MF, Raîche M, Gueye N'DR, Hébert R. Meeting the home-care needs of disabled older persons living in the community: does integrated services delivery make a difference? BMC Geriatrics 2011; 11:67.

47. Szebehely M, Trydegard GB. Home care for older people in Sweden: a universal model in transition. Health Soc Care Community 2012; 20(3):300-309.

48. Mayes PA, Silvers A, Prendergast JJ. New Direction for Enhancing Quality in Diabetes Care: Utilizing Telecommunications and Paraprofessional Outreach Workers Backed by an Expert Medical Team. Telemed J E Health. 2010; 16(3):358-363.

49. Golden AG, Roos BRDA, Silverman MA, Beers MH. Home and Community-Based Medicaid Options for Dependent older Floridians. J Am Geriatr Soc 2010; 58(2):371-376.

50. Russell D, Rosenfeld P, Ames S, Rosati RJ. Using Technology to Enhance the Quality of Home Health Care: Three Case Studies of Health Information Technology Initiatives at the Visiting Nurse Service of New York. $J$ Healthc Qual 2010; 32(5):22-29.

51. Seixas CT, Souza CG, Silva KL, Sena RR. Experiências de atenção domiciliar em Saúde no mundo: lições para o caso brasileiro. In: Brasil. Ministério da Saúde (MS). Atenção Domiciliar no SUS: resultados do laboratório de inovação em atenção domiciliar. Brasília: MS; 2014. p. 17-66.

52. Fripp JC, Facchini LA, Silva SM. Caracterização de um programa de internação domiciliar e cuidados paliativos no Município de Pelotas, Estado do Rio Grande do Sul, Brasil: uma contribuição à atenção integral aos usuários com câncer no Sistema Único de Saúde, SUS. Epidemiol Serv Saúde 2012; 21(1):69-78.

53. Merhy EE, Onocko R. Agir em Saúde: um desafio para o público. Sao Paulo: Hucitec Série Didática; 1997.

54. Marsteller JA, Burton L, Mader SL, Naughton B, Burl J, Guido S, Greenough WB, Steinwachs D, Clark R, Leff B. Health Care Provider Evaluation of a Substitutive Model of Hospital at Home. Med Care 2009; 47(9):979985.

55. Glendinning C. Home care in England: markets in the context of under-funding. Health Soc Care Community 2012; 20(3):292-299.

Artigo apresentado em 30/11/2014

Aprovado em 08/07/2015

Versão final apresentada em 10/07/2015 